\title{
Floquet-Engineered Vibrational Dynamics in a Two-Dimensional Array of Trapped Ions
}

\author{
Philip Kiefer®, ${ }^{1, *}$ Frederick Hakelberg $\odot,{ }^{1}$ Matthias Wittemer®, ${ }^{1}$ Alejandro Bermúdez, ${ }^{2, \dagger}$ \\ Diego Porras, ${ }^{3, \ddagger}$ Ulrich Warring, ${ }^{1}$ and Tobias Schaetz ${ }^{1}$ \\ ${ }^{1}$ Albert-Ludwigs-Universität Freiburg, Physikalisches Institut, Hermann-Herder-Strasse 3, 79104 Freiburg, Germany \\ ${ }^{2}$ Departamento de Física Teórica, Universidad Complutense, 28040 Madrid, Spain \\ ${ }^{3}$ Instituto de Física Fundamental IFF-CSIC, Calle Serrano 113b, 28006 Madrid, Spain
}

(Received 19 July 2019; published 21 November 2019)

\begin{abstract}
We demonstrate Floquet engineering in a basic yet scalable 2D architecture of individually trapped and controlled ions. Local parametric modulations of detuned trapping potentials steer the strength of longrange interion couplings and the related Peierls phase of the motional state. In our proof of principle, we initialize large coherent states and tune modulation parameters to control trajectories, directions, and interferences of the phonon flow. Our findings open a new pathway for future Floquet-based trapped-ion quantum simulators targeting correlated topological phenomena and dynamical gauge fields.
\end{abstract}

DOI: 10.1103/PhysRevLett.123.213605

A promising route for the exploration of complex quantum dynamics is to use experimental simulator devices where synthetic interactions and quantum states can be efficiently controlled [1]. In general, systems of interest should provide long-range interactions and spatial dimensions higher than 1 since these remain beyond the reach of numerical methods [2]. A variety of prototype platforms already exist [3]. Trapped atomic ions are a promising approach, featuring identical constituents, long-range Coulomb forces, and unique control of internal (electronic) and external (phonon) degrees of freedom [4,5]. Tremendous progress in common trapping potentials has been achieved [6,7]. Furthermore experiments have shown coupling of individual ions at distant sites by matching local motional frequencies in 1D [8-10] and in scalable 2D arrangements [11] with the perspective to preserve the unique control of one or a few ion ensembles. Typically, quantized vibrations (phonons) are used as auxiliary bus mediating entangling gate operations [12] or synthetic spinspin interactions [13]. In contrast, it has been proposed to actively use this degree of freedom. For example, to simulate complex bosonic lattice models [14] and to Floquet engineer an effective Peierls phase of the motional state, analogous to a synthetic gauge field $[15,16]$. In this context, phonons represent charged particles in external electromagnetic fields. They tunnel, and their trajectories enclose areas related to geometric phases or interfere directly between individually controlled ions, located at distinct sites of a dedicated lattice structure. A realization requires fine-tuning and parametric modulations of motional frequencies at each site. The strength of these drives tune the tunneling (coupling) strength, while control of the relative phases controls the accumulated Peierls phase. Such modulations enable interion couplings between detuned (decoupled) trapping potentials by absorption and emission of energy (photons or phonons) out of the classical driving field. Certain aspects of Floquet engineering by periodic modulations [17] have already been demonstrated for ultracold atoms [18-20], superconducting qubits [21], and photonic lattices [22].

In this Letter, we show essential features of phonon assisted coupling of individual atomic ions trapped at microsites of our triangular two-dimensional trap array. Parametric drives applied to single or multiple locations steer constructive and destructive coherent couplings within the array. Tuning driving amplitudes and relative phases, we control directionality and interference of the phonon flow via the related synthetic Peierls phase of the motional states, a key requirement for future quantum Floquet engineering.

We trap magnesium ions in our surface-electrode trap array featuring separate microsites $T_{\mathbf{j}}$, where $\mathbf{j} \in\{0,1,2\}$ labels the corners of the triangle with side lengths of $40 \mu \mathrm{m}$ and an ion-surface distance of $\simeq 40 \mu \mathrm{m}[23,24]$. In harmonic approximation, these distances yield an intersite coupling strength $\Omega_{C} /(2 \pi) \simeq 1 \mathrm{kHz}$ for motional frequencies $\omega_{\mathbf{j}} /(2 \pi) \simeq 4 \mathrm{MHz}$. Heating rates of (1-10) quanta/ms are derived from calibration measurements close to the motional ground state $[29,30]$. In order to receive unambiguous signals of the phonon dynamics, we initialize coherent states (exceeding $\simeq 1000$ motional quanta). The effective coupling rate $\Omega_{C \text {,eff }}$ is tunable via relative motional mode orientations and the detuning of the individual trapping sites. Anharmonic contributions of the trapping potential lead to an increased $\Omega_{C \text {,eff }} /(2 \pi) \simeq 6 \mathrm{kHz}$, while the related efficiency is reduced accordingly [11]. Quasistatic control potentials locally tune electric fields, curvatures, and higher order terms, e.g., for preparation or detection or 
intersite couplings [11]. We adiabatically ramp these control potentials within $t_{\text {ramp }} \leq 100 \mu \mathrm{s}\left[t_{\text {ramp }} \geq\left(\omega_{\mathbf{j}}\right)^{-1}\right]$ between different configurations. Additionally, we can apply various local periodic control potentials $V_{\mathbf{j}}^{\text {osc }}\left(\Omega_{\mathbf{j}}^{\text {osc }}, \varphi_{\mathbf{j}}^{\text {osc }}, u_{\mathbf{j}}^{\text {osc }}\right)$ for duration $t_{\mathbf{j}}^{\text {osc }}$, oscillating with frequency $\Omega_{\mathbf{j}}^{\text {osc }}$ with a tunable phase $\varphi_{\mathbf{j}}^{\text {osc }}$ and amplitude $u_{\mathbf{j}}^{\text {osc }}$, allowing for (i) Floquet engineering by a parametric modulation $V_{\mathbf{j}}^{\mathcal{M}}$ of motional frequencies with $\Omega_{\mathbf{j}}^{\mathcal{M}} /(2 \pi) \simeq 100 \mathrm{kHz}$ or (ii) initialization of a vibronic coherent state at a given $T_{\mathrm{j}}$ by a local excitation via $V_{\mathbf{j}}^{\mathcal{E}}$. All experiments are initialized by global Doppler cooling, aligning the local mode orientations, and tuning the lowest motional frequencies to $\omega_{\mathbf{j}} /(2 \pi) \simeq(3-5) \mathrm{MHz}$ $[11,23]$. In the following steps (see below), we apply dedicated control potentials for individual settings, and we finish with local fluorescence detection, allowing us to derive average phonon numbers $\bar{n}_{\mathbf{j}}$. The reduction of the fluorescence rate allows us to derive the increase of $\bar{n}_{\mathbf{j}}$ in a range of (100-10 000) quanta [29]. Each sequence is repeated 200 to 400 times to derive the standard error of the mean (s.e.m.).

To calibrate Floquet engineering via oscillating potentials $V_{\mathbf{j}}^{\mathcal{M}}$ (see the Supplemental Material [24]), we perform measurements with single ions at $T_{\mathbf{j}}$. Exemplarily, we discuss results for site $T_{1}$ (see Fig. 1), where we probe the effect of $V_{1}^{\mathcal{M}}\left(\Omega_{1}^{\mathcal{M}}, u_{1}^{\mathcal{M}}\right)$ for fixed $\Omega_{1}^{\mathcal{M}} /(2 \pi)=$ $100 \mathrm{kHz} \ll \omega_{1} /(2 \pi)$ with a simultaneously applied drive for coherent excitation $V_{1}^{\mathcal{E}}\left(\Omega_{1}^{\mathcal{E}}, u_{1}^{\mathcal{E}}\right)$. Tuning $\Omega_{1}^{\mathcal{E}}$ across $\omega_{1}$, we show reconstructed motional amplitudes for $u_{1}^{\mathcal{M}}=0 \mathrm{~V}$ [Fig. 1(b), top panel], $150 \mathrm{mV}$ [Fig. 1(b), middle panel], and $250 \mathrm{mV}$ [Fig. 1(b), bottom panel] depending on $\Omega_{1}^{\mathcal{E}}$. When $V_{1}^{\mathcal{M}}$ is switched on, a comb structure is spanned by several channels (sidebands) at $\Omega_{1}^{\mathcal{E}} \simeq \omega_{1}+m \Omega_{1}^{\mathcal{M}}$ with $m \in \mathbb{Z}$. Floquet theory (solid lines) predicts that the relative strength of these channels is defined by the $m$ th order Bessel function of the first kind $\mathcal{J}_{m}\left(\eta_{\mathbf{j}}\right)$, where $\eta_{\mathbf{j}} \propto u_{\mathbf{j}}^{\mathcal{M}} / \Omega_{\mathbf{j}}^{\mathcal{M}}$ represents the modulation index [24]. For increasing $u_{1}^{\mathcal{M}}$ [Fig. 1(b), middle and bottom panels], the carrier channel decreases until $\mathcal{J}_{0}\left(\eta_{\mathbf{j}}\right)$ crosses zero. This channel gets effectively shut and can lead in following experiments where we couple neighboring sites to so-called coherent destruction of tunneling [31]. Overall, we find $\eta_{\mathbf{j}} \Omega_{\mathbf{j}}^{\mathcal{M}} /(2 \pi)$ of up to $300 \mathrm{kHz}$ for $\Omega_{\mathbf{j}}^{\mathcal{M}} /(2 \pi) \simeq(50-200) \mathrm{kHz}$.

To demonstrate control of synthetic Peierls phases imprinted on the motional state in real time, we explore the assisted transfer of energy, i.e., the flow of phonons, between ions at different $T_{\mathbf{j}}$. As an example, we perform our experiment with single ions at $T_{0}$ and $T_{1}$; see Fig. 2. We adjust the intersite detuning to $\Delta \omega_{01} /(2 \pi)=$ $\left(\omega_{1}-\omega_{0}\right) /(2 \pi) \simeq 100 \mathrm{kHz}$ and excite the ion at $T_{0}\left(V_{0}^{\mathcal{E}}\right.$ for $\left.t_{0}^{\mathcal{E}}=20 \mu \mathrm{s}\right)$ to $\bar{n}_{0}=(5000-10000)$. By choice of $\Delta \omega_{01}$, the coupling efficiency (for $\eta_{\mathbf{j}}=0$ ) is suppressed by 4

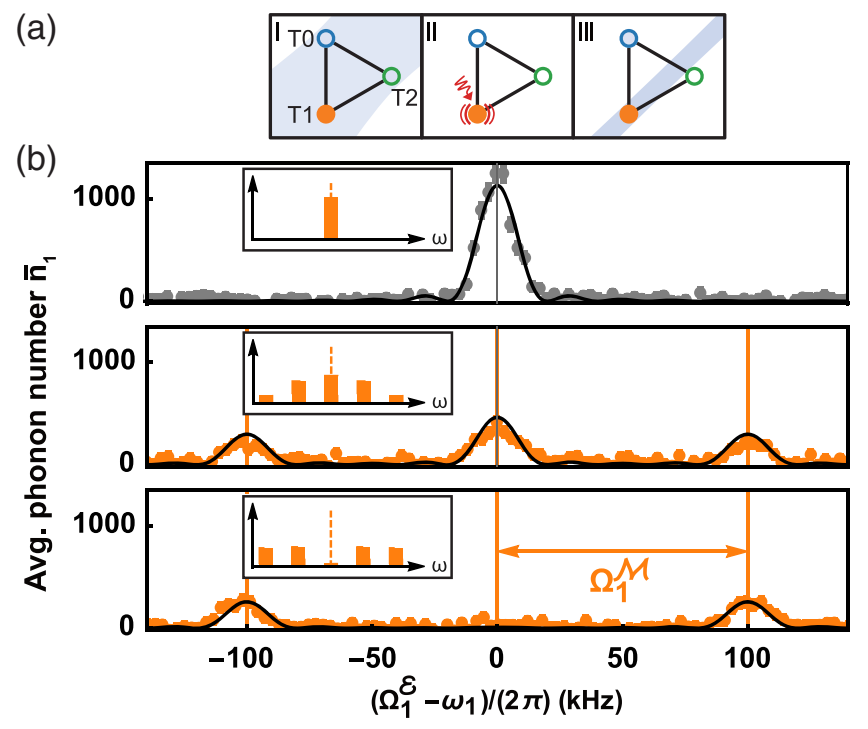

FIG. 1. Characterization of the ion's frequency spectrum during modulation at a single site. (a) Experimental sequence to characterize the effect of the modulation at $T_{1}$ on the motional eigenfrequency $\omega_{1}$ and its sidebands. These sidebands are interpreted as channels that, in the case of spectral overlap, can permit transmission between neighboring $T_{\mathbf{j}}$. The three equidistant trapping sites are marked by colored circles, the presence of an ion by a disk. (I) A single ion at $T_{1}$ is prepared via laser cooling (the shaded area). (II) The effect of the modulation potential $V_{1}^{\mathcal{M}}$ with variable strength (red brackets) is probed simultaneously via the excitation field $V_{1}^{\mathcal{E}}\left(\Omega_{1}^{\mathcal{E}}\right)$ (red wiggled arrow) for duration $t_{1}^{\mathcal{E}}=t_{1}^{\mathcal{M}}=50 \mu \mathrm{s}$. (III) Local detection of fluorescence from $T_{1}$ enables reconstruction of the ion's motional amplitude. (b) Final motional amplitudes (data points, error bars s.e.m.) depending on excitation frequency $\Omega_{1}^{\mathcal{E}}$ : (top panel) no modulation and (middle and bottom panels) increasing modulation amplitude. Model fits to the data (solid lines; see the Supplemental Material [24]) allow us to calibrate the reference coherent excitation, i.e., (top panel) $\eta_{1}=0$ and (middle and bottom panels) modulation indices with statistic uncertainties $\eta_{1}=1.26(3), 2.57(4)$. (Insets) Bar charts illustrate the derived amplitudes for the equally spaced spectral channels, shown up to the second sidebands. In this way, we can, e.g., strongly suppress excitations at the local oscillator frequency $\omega_{1}$.

orders of magnitude. Setting $\Omega_{1}^{\mathcal{M}}=\Delta \omega_{01}, \eta_{1} \simeq 1.8$, at a fixed $\varphi_{1}^{\mathcal{M}}$, the phonon exchange is enabled by assistance of $V_{1}^{\mathcal{M}}$ : a single transmission channel is opened by overlapping the lower first sideband at $T_{1}$ with the carrier at $\omega_{0}$ of $T_{0}$; see the Fig. 2(b) inset. In Fig. 2(b), we show the reconstructed $\bar{n}_{1}$ as a function of $t_{1}^{\mathcal{M}}$. We model the coherent exchange, absorption, and emission of the phonons, with the corresponding assisted coupling rate $\Omega_{\mathrm{AC}}$ (solid line; see the Supplemental Material [24]). We thus confirm that the ion at $T_{1}$ absorbs up to $\simeq 820$ phonons after $t_{\pi}=\pi / \Omega_{\mathrm{AC}}$. The efficiency is limited to about $15 \%$ of $\bar{n}_{0}$. We attribute that to the anharmonicity of the trapping potential probed by the currently large $\bar{n}_{1}$ [11]. 

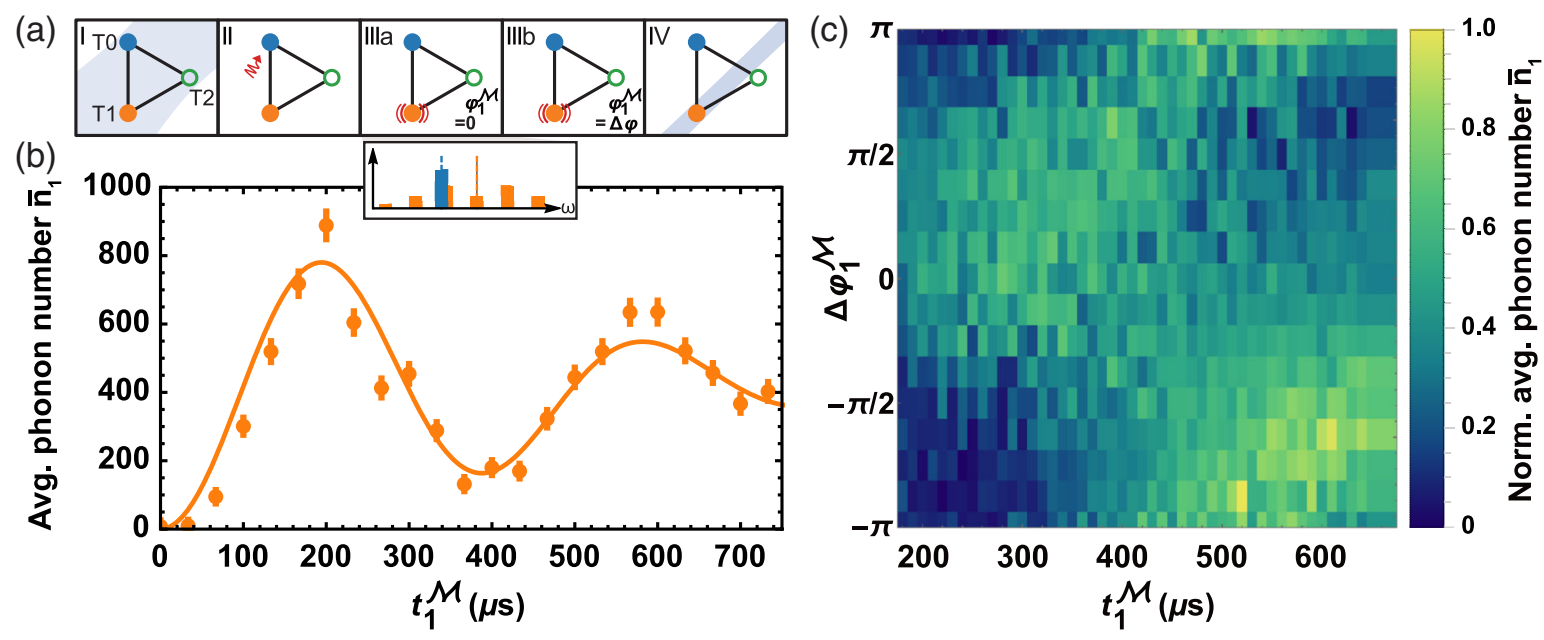

FIG. 2. Intersite coupling assisted by single-site modulation, enabling the control of directionality of the phonon flow. (a) Experimental sequence. (I) Initializing single ions at $T_{0}$ and $T_{1}$. (II) Switching to detuned coupling configuration and exciting the ion at $T_{0}$. (IIIa) Launching intersite coupling by application of the periodic modulation $V_{1}^{\mathcal{M}}$. (IIIb) To optionally redirect the phonon flow after $150 \mu \mathrm{s}$, we change the phase of $V_{1}^{\mathcal{M}}$. Here, a phase change by $\Delta \varphi_{1}^{\mathcal{M}}$ is realized within $25 \mu \mathrm{s}$, while $V_{1}^{\mathcal{M}}$ is active in total for $t_{1}^{\mathcal{M}}$. (IV) Detecting the motional amplitude $\bar{n}_{1}$. (b) Motional amplitude at $T_{1}$ as a function of $t_{1}^{\mathcal{M}}$ (data points, error bars s.e.m.) using part (IIIa). We choose $V_{1}^{\mathcal{M}}$ to open efficiently the transmission channel of the first lower sideband at $T_{1}$ (inset, orange bar chart) and the carrier at $T_{0}$ (blue bar, slightly shifted for visibility). Phonons are coherently exchanged between $T_{0}$ and $T_{1}$. A model fit (solid line) yields an assisted coupling rate $\Omega_{\mathrm{AC}} /(2 \pi)=2.51(5) \mathrm{kHz}$ and dephasing duration of $\tau=550(60) \mu \mathrm{s}$. (c) Normalized motional amplitude depending on $t_{1}^{\mathcal{M}}$ and $\Delta \varphi_{1}^{\mathcal{M}}$ including sequence part (IIIb). Real-time control of the Peierls phase $\Phi^{P}(t)$ and the related directionality of the phonon flow are set by $\Delta \varphi_{1}^{\mathcal{M}}$.

The anharmonic effects can be interpreted as additional detuning, increasing $\Omega_{\mathrm{AC}}$ but limiting efficiency. Working at the level of single quanta will reduce $\Omega_{\mathrm{AC}}$ to $\simeq 2 \pi \times$ $1 \mathrm{kHz}$ but increase the phonon exchange efficiency.

The Peierls phase $\Phi^{P}(t)$ plays an important role in the Floquet-engineered Hamiltonian of energy transfer between different sites [24]. It is given by the path integral along $l$ between trapping sites $\Phi^{P}(t)=(q / \hbar) \int_{T_{\mathbf{j}}}^{T_{\mathrm{i}}} d \boldsymbol{l} \cdot \boldsymbol{A}(\boldsymbol{r}, t)$, where $\hbar$ is the reduced Planck constant. $\Phi^{P}$ rules the dynamics of the phonons as if they were particles with charge $q$ coupled to a gauge potential $\boldsymbol{A}(\boldsymbol{r}, t)$. In an extended experimental sequence, the evolution of $\Phi^{P}$ is controlled in real time during the experiment. In particular, after a period of assisted coupling of $t_{1 \text {,prep }}^{\mathcal{M}}=t_{\pi} / 2 \simeq 150 \mu \mathrm{s}$, we adiabatically $\operatorname{ramp} \varphi_{1}^{\mathcal{M}} \rightarrow \varphi_{1}^{\mathcal{M}}+\Delta \varphi_{1}^{\mathcal{M}}$ within $t_{\varphi, \text { ramp }}=$ $25 \mu \mathrm{s} \gg \omega_{\mathrm{j}}^{-1}$ and continue the modulation for duration $t_{1}^{\mathcal{M}}-\left(t_{1, \text { prep }}^{\mathcal{M}}+t_{\varphi, \text { ramp }}\right)$. We depict normalized $\bar{n}_{1}$ as a function of $\Delta \varphi_{1}^{\mathcal{M}}$ and $t_{1}^{\mathcal{M}}$ in Fig. 2(c). The dependence of the number of exchanged phonons on $\Delta \varphi_{1}^{\mathcal{M}}$ shows that $\Phi^{P}$ cannot be simply gauged away for $\Delta \varphi_{1}^{\mathcal{M}} \neq 0$ but, instead, serves to control the directionality of the coherent energy flow. At $t_{1}^{\mathcal{M}} \simeq 300 \mu \mathrm{s}$, the optimal exchange is observed for $\Delta \varphi_{1}^{\mathcal{M}} \simeq+\pi / 4$. For $\Delta \varphi_{1}^{\mathcal{M}} \approx-3 \pi / 4$, a change by $\pi$, we observe the transferred population nearly vanishing. This is consistent with Floquet theory, which predicts that $\Omega_{\mathrm{AC}}\left(\varphi_{1}^{\mathcal{M}}\right) \rightarrow-\Omega_{\mathrm{AC}}\left(\varphi_{1}^{\mathcal{M}}+\pi\right)$, equivalent to a time-reversal operation. That is, it returns phonons from $T_{1}$ back to $T_{0}$ and further simulates the application of an electric field on charged particles. In particular, $\partial \Phi^{P}(t) / \partial t \neq 0$ can be understood as a background synthetic electric field $\boldsymbol{E}(\boldsymbol{r}, t)=-\partial \boldsymbol{A}(\boldsymbol{r}, t) / \partial t$. We observe a global shift by $\pi / 4$ in the data. Numerical simulations can provide evidence for a similar shift when considering a mismatch between $\Omega_{1}^{\mathcal{M}}$ and $\Delta \omega_{01}$ of a few percent and the finite ramping duration $t_{\varphi, \text { ramp }}$.

In the next sequence, we explore the dynamics between $T_{0}$ and $T_{1}$ when both sites are locally driven by $V_{0}^{\mathcal{M}}$ and $V_{1}^{\mathcal{M}}$ at fixed $\Omega_{0}^{\mathcal{M}}=\Omega_{1}^{\mathcal{M}} \simeq \Delta \omega_{01}$ and $\eta_{0} \simeq \eta_{1}$. In this case, the assisted exchange can be controlled by the relative modulation phase $\Delta \varphi_{01}^{\mathcal{M}}=\varphi_{0}^{\mathcal{M}}-\varphi_{1}^{\mathcal{M}}$, here reaching $\Omega_{\mathrm{AC}} /(2 \pi) \leq 4.5 \mathrm{kHz}$ [11]. As shown in the insets of Figs. 3(b) and 3(c), we open several transmission channels, and the overall phonon exchange is governed by constructive or destructive interference of all contributions as a function of $\Delta \varphi_{01}^{\mathcal{M}}$. We show the results of $\bar{n}_{1}$ after $t_{01}^{\mathcal{M}} \simeq t_{\pi}$ for $\eta_{0} \simeq \eta_{1} \simeq \eta \simeq\{0.9,1.7\}$ in Figs. 3(b) and 3(c), respectively. For $\eta \simeq 0.9$, transmission is predominantly enabled by the resonance of two distinct channels, corresponding to the carriers and first sidebands at $T_{0}$ and $T_{1}$; see the Fig. 3(b) inset. As shown in Fig. 3(b), the measured data are consistent with the Floquet-engineered $\Omega_{\mathrm{AC}}$, considering a linear coupling between harmonic oscillators (the dashed line) [16]: $\Omega_{\mathrm{AC}}$ is significantly suppressed for $\Delta \varphi_{01}^{\mathcal{M}}=0$ 


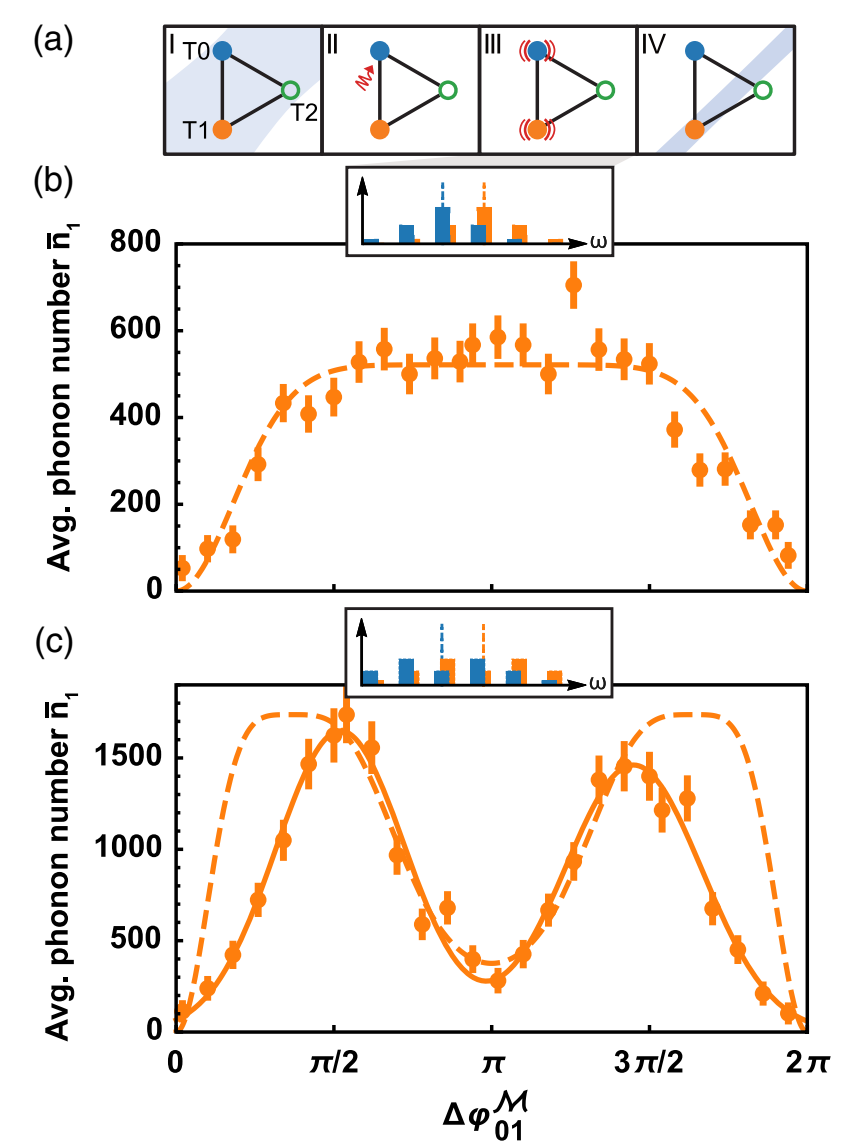

FIG. 3. Tunable assisted coupling, established by selection of transmission channels and its dependence on the relative phase of two modulations. (a) Experimental sequence. (I) Preparation of single ions at $T_{0}$ and $T_{1}$. (II) Initialization of phonons at $T_{0}$ by application of $V^{\mathcal{E}}$ at detuned intersite coupling configuration. (III) Activation of assisted coupling by $V_{0}^{\mathcal{M}}$ and $V_{1}^{\mathcal{M}}\left(\Delta \varphi_{01}^{\mathcal{M}}\right)$ with $\eta \simeq$ $\eta_{0} \simeq \eta_{1}$ for $t^{\mathcal{M}}=t_{\pi}$, i.e., optimized exchange. (IV) Detection at $T_{1}$. (b),(c) Motional amplitude (data points, error bars s.e.m.) depending on $\Delta \varphi_{01}^{\mathcal{M}}$ for (b) $\eta \simeq 0.9$ and (c) $\eta \simeq 1.7$. (Insets) Illustration of multiple transmission channels that mutually interfere and provide the effective coupling. The idealized, scaled model (dashed lines) describes the dependency qualitatively well, while it lacks the ability to explain systematics in (c) outside the central region (the solid line is a guide for the eye); see the text. Fundamentally, the energy transfer is a consequence of interferences of all transmission channels and is tuned by $\Delta \varphi_{01}^{\mathcal{M}}$ at $\eta_{0} \simeq \eta_{1}$.

and $2 \pi$, while the transfer to $T_{1}$ is maximal and robust around $\Delta \varphi_{01}^{\mathcal{M}}=\pi$. In the first case, both ions are modulated with the same phase; thus their exchange of vibrational excitation cannot be activated by absorbing energy from the drive. That is, the driving Hamiltonian commutes with the coupling terms. A residual coupling can be explained by a residual mismatch of the modulation indices $\left(\eta_{0} \neq \eta_{1}\right)$ and intersite dephasing. In the latter case, the activation of the coupling via the two channels leads to the flat plateau. Stronger modulation [see the Fig. 3(c) inset] opens (a)

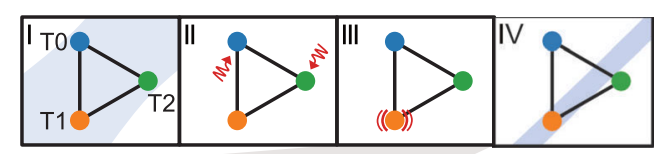

(b)
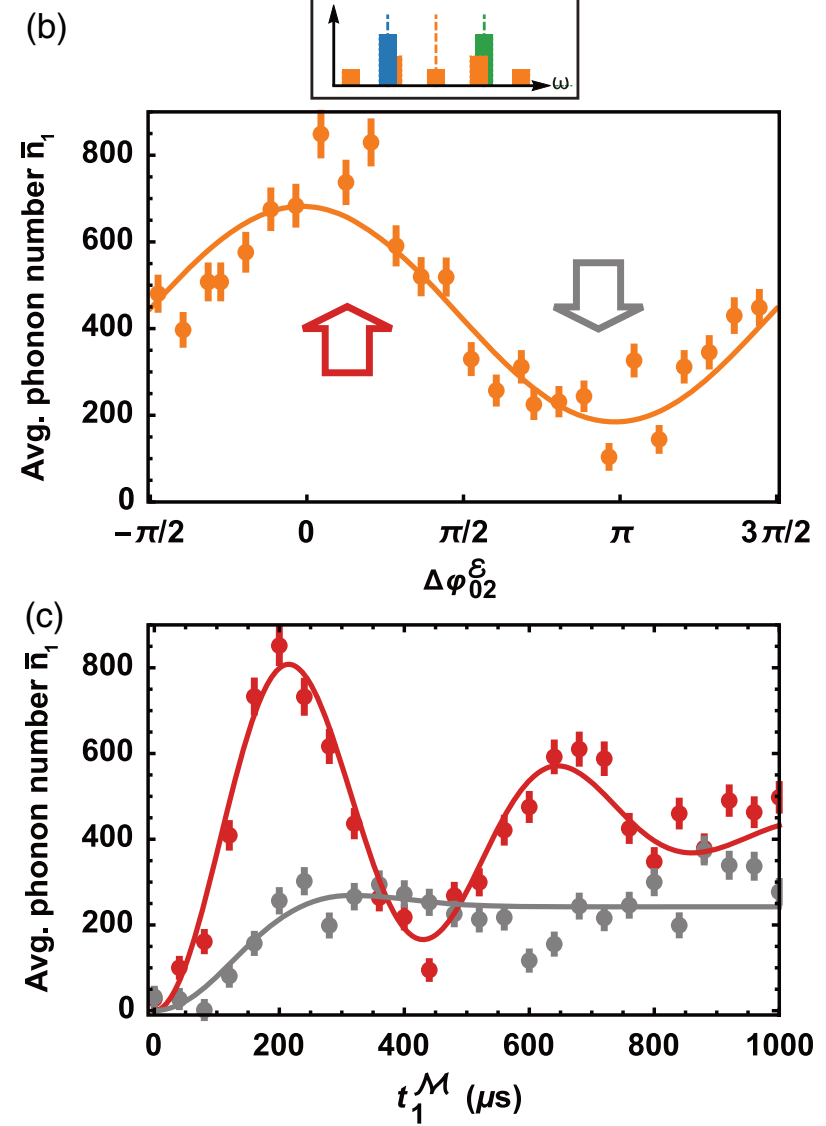

FIG. 4. Controlled interference within a modulation-assisted 2D network of coupled oscillators. (a) Experimental sequence. (I) Global preparation of single ions at $T_{\mathrm{j}}$. (II) Coupled network configuration offers a local gradient between the individual trapping sites $\Delta \omega_{01}=\Delta \omega_{12}$. Two local excitation pulses control the relative phase between coherent states at $T_{0}$ and $T_{2}$. (III) Coupling the network via modulation $V_{1}^{\mathcal{M}}$ for $t_{1}^{\mathcal{M}}$. (IV) Detection at $T_{1}$. (b, top panel) Modulation $V_{1}^{\mathcal{M}}$ opens transmission channels between $T_{0}$ and $T_{1}$ as well as between $T_{1}$ and $T_{2}$. (b, bottom panel) Average phonon number $\bar{n}_{1}$ as a function of $\Delta \varphi_{02}^{\mathcal{E}}$ for $t^{\mathcal{M}}=200 \mu$ s (data points, error bars s.e.m.). The orange line represents a sinusoidal model fit yielding an amplitude of 250(20) quanta and an offset of 430(20) quanta. (c) Evolution for characteristic constructive (red) and destructive (gray) interference within the network. Model fits (solid lines) depict suppressed $\Omega_{\mathrm{AC}}$ in the destructive case and dephasing durations of $620(50) \mu \mathrm{s}$ (red) and $250(80) \mu \mathrm{s}$ (gray). Phase-dependent dephasing durations as well as residual oscillations in the destructive case will be further investigated.

additional channels; i.e., owing to the corresponding Bessel functions, this leads to a larger contribution of the upper and lower sidebands. In Fig. 3(c), the data show additional features of the phase-dependent energy transfer, e.g., an additional destructive interference near $\Delta \varphi_{01}^{\mathcal{M}}=\pi$, 
in accordance with the prediction. We note, however, that the two peaks of maximal phonon exchange are narrowed with respect to the idealized theory, and a slight asymmetry appears, which we attribute to anharmonicities resulting from the Coulomb interaction, as well as local trapping potentials.

To demonstrate interference of phonons in two dimensions, a key feature to simulate Aharonov-Bohm effects of charged particles subjected to a vector potential, all $T_{\mathbf{j}}$ are initialized. We prepare for multisite coupling by tuning $\omega_{\{0,1,2\}} /(2 \pi) \simeq\{4.9,5.0,5.1\}$ MHz. We simultaneously apply $V_{0}^{\mathcal{E}}\left(\varphi_{0}^{\mathcal{E}}\right)$ and $V_{2}^{\mathcal{E}}\left(\varphi_{2}^{\mathcal{E}}\right)$ to prepare coherent states with $\bar{n}_{0} \simeq 5500$ and $\bar{n}_{2} \simeq 5800$ phonons and, importantly, a fixed phase relation $\Delta \varphi_{02}^{\mathcal{E}}$ [32]. Multisite phonon coupling is activated by applying $V_{1}^{\mathcal{M}}$ with $\Omega_{1}^{\mathcal{M}} \simeq \Delta \omega_{01} \simeq \Delta \omega_{12}$ and $\eta_{1} \simeq 1.8$ during $t_{1}^{\mathcal{M}}$. The lower and upper first sidebands at $T_{1}$ open transmission channels with the carrier at sites $T_{0}$ and $T_{2}$, respectively; see Fig. 4(b), top panel. We note that direct phonon exchange between $T_{0}$ and $T_{2}$ is disabled by the frequency mismatch $\Delta \omega_{02} \gg \Omega_{\mathrm{AC}}$. The results in Fig. 4(b) depict $\bar{n}_{1}$ as a function of $\Delta \varphi_{02}^{\mathcal{E}}$ for the maximal exchange achieved at $t_{1}^{\mathcal{M}}=t_{\pi}$. While the energy transfer is maximal at $\Delta \varphi_{02}^{\mathcal{E}}=0$ (the constructive interference highlighted by the red arrow), it is minimal at $\Delta \varphi_{02}^{\mathcal{E}}=\pi$ (the destructive interference highlighted by the gray arrow). In Fig. 4(c), we investigate both of the extremal settings depending on $t_{1}^{\mathcal{M}}$, depicting the coherent destruction of energy transfer in 2D.

To summarize, we demonstrate in this Letter Floquet engineering of vibrational excitations in a $2 \mathrm{D}$ ion-trap array, present clear signatures of interference effects, and discuss the role of the arising dynamical Peierls phase. In future studies, both argon-ion bombardment [33] or cryogenic environments [34] can reduce heating rates by more than 2 orders of magnitude, permitting operation near the motional ground state for durations $\gg 1 / \Omega_{\mathrm{AC}}$, as established for short timescales already [29]. Furthermore triangular lattices, plaquettes to concatenate rhombic ladders, and even more complex, nonperiodic structures can be realized in future arrays [35,36]. Combining these techniques with the presented Floquet toolbox may additionally enable us to study the interplay of nonlinearities, i.e., effective on-site phonon-phonon interactions, with the synthetic gauge fields. This would enable us to explore correlated, symmetry-protected topological phases of bosons [37]. By exploiting laser cooling and heating mechanisms [38], we can build a phononic analog of photonic lattices [39-42] with a rich interplay between topological and dissipative effects. Application of statedependent optical potentials may further extend the quantum-simulation prospects of our platform by enabling state-dependent transmission. In particular, by coupling the internal degrees of freedom to the vibrations, one may study bosonic lattice models in the presence of dynamical gauge fields, e.g., famous Aharonov-Bohm physics on closed cycles, cages, and edge states $[16,43]$.

We thank J.-P. Schröder for the help with the experimental control system. The trap chip was designed in collaboration with R. Schmied in cooperation with the NIST ion storage group and produced by Sandia National Laboratories. This work was supported by the Deutsche Forschungsgemeinschaft (DFG) (Grant No. SCHA 973/ 6-3) and the Georg H. Endress foundation. A. B. acknowledges support from the Ramón y Cajal Program RYC-2016-20066, CAM/FEDER Project S2018/TCS4342 (QUITEMAD-CM), and PGC2018-099169-B-I00 (MCIU/AEI/FEDER, UE). D. P. acknowledges support from PGC2018-094792-B-I00 (MCIU/AEI/FEDER, UE).

P. K. and F. H. contributed equally to this work.

*philip.kiefer@physik.uni-freiburg.de; https://www.qsim .uni-freiburg.de

†albermud@ucm.es

td.porras@iff.csic.es

[1] J. I. Cirac and P. Zoller, Nat. Phys. 8, 264 (2012).

[2] F. Verstraete, J. I. Cirac, and V. Murg, Adv. Phys. 57, 143 (2008).

[3] I. M. Georgescu, S. Ashhab, and F. Nori, Rev. Mod. Phys. 86, 153 (2014).

[4] C. J. Ballance, T. P. Harty, N. M. Linke, M. A. Sepiol, and D. M. Lucas, Phys. Rev. Lett. 117, 060504 (2016).

[5] J. P. Gaebler, T. R. Tan, Y. Lin, Y. Wan, R. Bowler, A. C. Keith, S. Glancy, K. Coakley, E. Knill, D. Leibfried, and D. J. Wineland, Phys. Rev. Lett. 117, 060505 (2016).

[6] J. Zhang, G. Pagano, P. W. Hess, A. Kyprianidis, P. Becker, H. Kaplan, A. V. Gorshkov, Z.-X. Gong, and C. Monroe, Nature (London) 551, 601 (2017).

[7] E. Jordan, K. A. Gilmore, A. Shankar, A. Safavi-Naini, J. G. Bohnet, M. J. Holland, and J. J. Bollinger, Phys. Rev. Lett. 122, 053603 (2019).

[8] K. R. Brown, C. Ospelkaus, Y. Colombe, A. C. Wilson, D. Leibfried, and D. J. Wineland, Nature (London) 471, 196 (2011).

[9] M. Harlander, R. Lechner, M. Brownnutt, R. Blatt, and W. Hänsel, Nature (London) 471, 200 (2011).

[10] A. C. Wilson, Y. Colombe, K. R. Brown, E. Knill, D. Leibfried, and D. J. Wineland, Nature (London) 512, 57 (2014).

[11] F. Hakelberg, P. Kiefer, M. Wittemer, U. Warring, and T. Schaetz, Phys. Rev. Lett. 123, 100504 (2019).

[12] V. M. Schäfer, C. J. Ballance, K. Thirumalai, L. J. Stephenson, T. G. Ballance, A. M. Steane, and D. M. Lucas, Nature (London) 555, 75 (2018).

[13] H. Schmitz, A. Friedenauer, C. Schneider, R. Matjeschk, M. Enderlein, T. Huber, J. Glueckert, D. Porras, and T. Schaetz, Appl. Phys. B 95, 195 (2009).

[14] D. Porras and J. I. Cirac, Phys. Rev. Lett. 92, 207901 (2004).

[15] A. Bermudez, T. Schaetz, and D. Porras, Phys. Rev. Lett. 107, 150501 (2011). 
[16] A. Bermudez, T. Schaetz, and D. Porras, New J. Phys. 14, 053049 (2012).

[17] A. Eckardt, Rev. Mod. Phys. 89, 011004 (2017).

[18] M. Aidelsburger, M. Atala, S. Nascimbène, S. Trotzky, Y.-A. Chen, and I. Bloch, Phys. Rev. Lett. 107, 255301 (2011).

[19] J. Struck, C. Ölschläger, M. Weinberg, P. Hauke, J. Simonet, A. Eckardt, M. Lewenstein, K. Sengstock, and P. Windpassinger, Phys. Rev. Lett. 108, 225304 (2012).

[20] L. Asteria, D. T. Tran, T. Ozawa, M. Tarnowski, B. S. Rem, N. Fläschner, K. Sengstock, N. Goldman, and C. Weitenberg, Nat. Phys. 15, 449 (2019).

[21] P. Roushan et al., Nat. Phys. 13, 146 (2017).

[22] S. Mukherjee, M. Di Liberto, P. Öhberg, R. R. Thomson, and N. Goldman, Phys. Rev. Lett. 121, 075502 (2018).

[23] M. Mielenz, H. Kalis, M. Wittemer, F. Hakelberg, U. Warring, R. Schmied, M. Blain, P. Maunz, D. L. Moehring, D. Leibfried, and T. Schaetz, Nat. Commun. 7, ncomms11839 (2016).

[24] See Supplemental Material at http://link.aps.org/ supplemental/10.1103/PhysRevLett.123.213605 for details on the experimental setup, methods and vibrational dynamics, which includes Refs. [25-28].

[25] R. Schmied, J. H. Wesenberg, and D. Leibfried, Phys. Rev. Lett. 102, 233002 (2009).

[26] R. Bowler, U. Warring, J. W. Britton, B. C. Sawyer, and J. Amini, Rev. Sci. Instrum. 84, 033108 (2013).

[27] K. G. Johnson, J. D. Wong-Campos, A. Restelli, K. A. Landsman, B. Neyenhuis, J. Mizrahi, and C. Monroe, Rev. Sci. Instrum. 87, 053110 (2016).

[28] H. Kalis, F. Hakelberg, M. Wittemer, M. Mielenz, U. Warring, and T. Schaetz, Phys. Rev. A 94, 023401 (2016).

[29] H. Kalis, Ph. D. thesis, University of Freiburg, 2017.
[30] A. Friedenauer, F. Markert, H. Schmitz, L. Petersen, S. Kahra, M. Herrmann, T. Udem, T. Hänsch, and T. Schätz, Appl. Phys. B 84, 371 (2006).

[31] M. Grifoni and P. Haenggi, Phys. Rep. 304, 229 (1998).

[32] Note that the phase relation of the ion oscillators results from different constant phase offsets, caused, e.g., by supply wiring of the control electrodes, anharmonic contributions of the trapping potential, or the duration between the start of excitation and modulation potentials.

[33] D. A. Hite, Y. Colombe, A. C. Wilson, K. R. Brown, U. Warring, R. Jördens, J. D. Jost, K. S. McKay, D. P. Pappas, D. Leibfried, and D. J. Wineland, Phys. Rev. Lett. 109, 103001 (2012).

[34] J. Labaziewicz, Y. Ge, P. Antohi, D. Leibrandt, K. R. Brown, and I. L. Chuang, Phys. Rev. Lett. 100, 013001 (2008).

[35] T. Schaetz, A. Friedenauer, H. Schmitz, L. Petersen, and S. Kahra, J. Mod. Opt. 54, 2317 (2007).

[36] T. Schaetz, C. R. Monroe, and T. Esslinger, New J. Phys. 15, 085009 (2013).

[37] S. D. Huber and N.H. Lindner, Proc. Natl. Acad. Sci. U.S.A. 108, 19925 (2011).

[38] A. Lemmer, C. Cormick, D. Tamascelli, T. Schaetz, S. F. Huelga, and M. B. Plenio, New J. Phys. 20, 073002 (2018).

[39] A. Bermudez, M. Bruderer, and M. B. Plenio, Phys. Rev. Lett. 111, 040601 (2013).

[40] V. Peano, M. Houde, F. Marquardt, and A. A. Clerk, Phys. Rev. X 6, 041026 (2016).

[41] T. Ozawa, H. M. Price, A. Amo, N. Goldman, M. Hafezi, L. Lu, M. C. Rechtsman, D. Schuster, J. Simon, O. Zilberberg, and I. Carusotto, Rev. Mod. Phys. 91, 015006 (2019).

[42] D. Porras and S. Fernández-Lorenzo, Phys. Rev. Lett. 122, 143901 (2019).

[43] A. Bermudez and D. Porras, New J. Phys. 17, 103021 (2015). 